\title{
COMUNICACIÓN EDUCATIVA Y CULTURA POlítica EN El Movimiento 15-M. Aproximación TEÓRICA Y REFLEXIONES PRELIMINARES EN TORNO A UN ESTUDIO ETNOGRÁFICO
}

Educational Communication and Political Culture in the 15-M Movement. Theoretical Approach and Preliminary Reflections about an Ethnographic Stud

\section{Ángel Barbas Coslado \\ Universidad Nacional de Educación a Distancia (UNED)}

RESUMEN: En este artículo, presentamos algunos aspectos de la investigación etnográfica que hemos realizado sobre la dimensión pedagógico-política que subyace en los entornos y en los procesos de comunicación del Movimiento 15-M. Concretamente, abordamos los fundamentos teóricos de nuestro estudio a partir de los conceptos de comunicación educativa y cultura política, y reflexionamos sobre el carácter pedagógico-político implícito tanto en las prácticas asamblearias como en los proyectos de comunicación que el Movimiento 15-M ha desarrollado desde su aparición.

Palabras Clave: movimiento 15-M, comunicación educativa, cultura política, prácticas asamblearias, proyectos de comunicación.

RESUM: En aquest article presentem alguns aspectes de la investigació etnogràfica que hem realitzat sobre la dimensió pedagogicopolítica que és subjacent als voltants $\mathrm{i}$ en els processos de comunicació del Moviment 15-M. Concretament, abordem els fonaments teòrics del nostre estudi a partir dels conceptes de comunicació educativa i cultura politica, i reflexionem sobre el caràcter pedagogicopolític implícit tant en les pràctiques assembleàries com 
en els projectes de comunicació que el Moviment 15-M ha desenvolupat des que va aparèixer.

Paraules clau: Moviment 15-M, comunicació educativa, cultura política, pràctiques assembleàries, projectes de comunicació.

ABSTRACT: In this paper, we present some aspects of the ethnographic research that we have done about the pedagogical-political dimension that underlies in the communication environments and processes of the 15-M movement. We specifically deal with the theoretical foundations of our study from the concepts of 'educational communication' and 'political culture', and we reflect on the pedagogical-political nature which is inherent in both the assembly-based practices and communication projects that the 15-M movement has developed since its emergence.

KEYWORDS: 15-M movement, educational communication, political culture, assembly-based practices, communication projects.

\section{Introducción}

$\mathrm{E}^{1}$

15 de mayo de 2011, varios colectivos organizados en torno a la Plataforma de Coordinación de Grupos Pro-movilización Ciudadana convocan una serie de manifestaciones en diferentes ciudades españolas con el objetivo de promover una democracia más participativa y reivindicar transformaciones políticas ante lo que se percibe como un sistema obsoleto e incapaz de dar respuestas a las demandas de la ciudadanía. Lo que en apariencia iba a ser otra manifestación más, como tantas otras que son olvidadas poco tiempo después, en días sucesivos se convierte en un conjunto de acciones que transforman las calles y las redes en laboratorios de pensamiento ciudadano, en espacios de deliberación donde se discuten propuestas alternativas y donde se construyen otros modelos de sociedad. Nació entonces el movimiento social que se popularizaría con el nombre de Movimiento 15-M. 
El 15-M se ha convertido en un fenómeno de máximo interés para las Ciencias Sociales. Encontramos trabajos que abordan el estudio del movimiento desde ámbitos académicos como la Comunicación, la Antropología, el Derecho, la Educación, la Filosofía, la Sociología y las Ciencias Políticas. El trabajo que aquí presentamos es parte de una investigación, enmarcada en el ámbito de la Comunicación Educativa, que pretende abordar el estudio de la dimensión pedagógico-política implícita en los proyectos de comunicación surgidos a raíz del movimiento. Concretamente, realizamos un estudio etnográfico en «Ágora Sol Radio» utilizando la observación participante y las entrevistas en profundidad como técnicas de recogida de datos.

La relevancia de esta investigación se justifica por la ausencia de trabajos que aborden el estudio del Movimiento 15-M desde la perspectiva de la Comunicación Educativa. Para conocer el estado de la cuestión, ha sido necesario realizar una exhaustiva revisión bibliográfica dividida en dos enfoques: por un lado, se ha revisado la literatura de la Comunicación Educativa para conocer de qué manera se está abordando este movimiento desde dicho campo de estudios y, por otro, se ha revisado la literatura sobre el 15-M prestando especial atención al tratamiento de las dimensiones mediático-comunicacional y pedagógico-política.

En cuanto a la primera, se ha comprobado que tanto los movimientos sociales, en general, como el Movimiento 15-M, en particular, han pasado prácticamente inadvertidos para los académicos y estudiosos del ámbito de la Comunicación Educativa que han publicado trabajos en los últimos años; algo que, a tenor del carácter comunicacional y educativo del movimiento, supone una importante laguna para este campo de estudios.

En cuanto a la segunda, hemos observado que la mayoría de los trabajos académicos plantean la comunicación como una herramienta que posibilita determinadas prácticas sociales; ya sea la transmisión de mensajes, la creación de espacios para el debate o la coordinación de actos de protesta. Por otra parte, aunque encontramos estudios donde se menciona la dimensión pedagógico-política del 15-M, éste es un aspecto que no está suficientemente abordado en la literatura específica sobre el movimiento.

Por consiguiente, en ningún caso se ha realizado un análisis comunicativo-educativo del Movimiento 15-M; es decir, un análisis que aborde el 
estudio de la comunicación como un proceso de construcción de sentido, como un proceso mediante el cual los participantes construyen nuevas estructuras simbólicas, nuevos esquemas de pensamiento y nuevos marcos de interpretación de la realidad; en síntesis, un análisis que aborde el estudio de la comunicación desarrollada por el Movimiento 15-M como una dinámica de producción cultural que es, implícitamente, pedagógica. Consideramos urgente investigar estos aspectos para comenzar a entender el verdadero alcance de las transformaciones que el 15-M podría estar generando; algo que, además, enriquecería significativamente el corpus teórico-práctico tanto de los estudios sobre comunicación y movimientos sociales como de los estudios del ámbito específico de la Comunicación Educativa en contextos no formales.

En este artículo, damos cuenta de algunos aspectos de esta investigación. Concretamente, abordaremos los conceptos de comunicación educativa y cultura politica para reflexionar en torno a la dimensión pedagógico-política implícita en las prácticas y en los procesos de comunicación que el Movimiento 15-M ha desarrollado desde su aparición. En primer lugar, nos detendremos en el concepto de comunicación educativa como marco y fundamento de nuestro trabajo; en segundo lugar, definiremos nuestro objeto de estudio desde la noción de cultura política; en tercer lugar, analizaremos la formación de la cultura política de la ciudadanía a partir de las prácticas asamblearias; y, finalmente, expondremos una serie de reflexiones finales en torno a los proyectos de comunicación del 15-M como entornos de comunicación educativa y de cultura política.

\section{La Comunicación Educativa, un marco pedagógico-político}

La Comunicación Educativa es un campo de estudios interdisciplinar y transdisciplinar que aborda la dimensión pedagógica implícita en los procesos y las prácticas comunicacionales. Habitualmente, es equiparada a nociones como comunicación/educación, educomunicación y educación mediática, entre otras (Barbas 2012); sin embargo, aunque todas expresan la relación interdependiente de los fenómenos comunicativos y educativos, cada una de ellas pone el énfasis en una dimensión específica. En el caso de comunica- 
ción/educación y educomunicación el énfasis está puesto en la articulación de la dimensión educativa y de la dimensión comunicacional en una misma herramienta conceptual. Por su parte, en el caso de la noción de educación mediática, el énfasis está puesto en los procesos de enseñanza-aprendizaje de, con y a través de los medios de comunicación y las TIC. En esta investigación utilizamos la noción de comunicación educativa para enmarcar y fundamentar el análisis de la dimensión pedagógico-política implícita en los procesos de producción de los medios de comunicación surgidos a raíz del Movimiento 15-M y lo hacemos argumentando dos razones principales.

En primer lugar, el concepto de comunicación educativa nos permite establecer una clara diferencia entre los medios como objetos de enseñanza y los medios como vehículos de aprendizaje. De este modo, hacemos referencia a las dinámicas de educación informal que se canalizan a través de los medios de comunicación; es decir, a los procesos de aprendizaje implícitos que las personas experimentan cuando participan en un proyecto de comunicación. Autores como Gutiérrez (1973 y 1975); Porcher (1974); Vallet (1977); Aparici y García Matilla (1987 y 2008); Masterman (1993); McQuail (1999) y Ferrés (2008 y 2014), entre otros, han estudiado la penetración de los mensajes de los medios de comunicación en la sociedad; su grado de influencia en la conformación de identidades colectivas, de imaginarios sociales, de esquemas de interpretación del mundo; su poder para favorecer estados de opinión así como para reforzar determinadas ideologías, deseos y valores; y han indicado la necesidad de impulsar la participación ciudadana en la producción mediática con el objetivo de favorecer el conocimiento de las estructuras y de los lenguajes de los medios de comunicación. Asimismo, como señala Kaplún (1992), es mediante los procesos de socialización y de comunicación en los que la ciudadanía se involucra, tanto dentro como fuera de las instituciones de educación formal y en todo tipo de dinámicas comunicacionales, cuando se aprende y cuando se adquiere el verdadero conocimiento; el autor expresa esta idea de la siguiente manera:

Así como resulta evidente que la comunicación de algo presupone el conocimiento de aquello que se comunica, no suele verse con la misma claridad que la inversa también se da: al pleno conocimiento de ese algo se llega cuando existe la ocasión y la exigencia de comunicarlo. Es en ese esfuerzo de socializa- 
ción cuando se va profundizando en el conocimiento a ser comunicado y descubriendo aspectos hasta entonces vagamente intuidos [...]. (p. 36)

En segundo lugar, utilizamos el concepto de comunicación educativa basándonos en su tradición epistemológica y, más concretamente, en su posicionamiento crítico y en su orientación hacia la transformación social y política. La comunicación educativa forma parte de la corriente de la comunicación crítica latinoamericana, un campo de estudios que surge en el contexto de las experiencias de comunicación popular y participativa que se desarrollaron en diferentes países de América Latina a partir de la década de los años 40 del siglo pasado. Los trabajos de autores como Freire (1970/2007), en primer lugar, y de Gutiérrez (1973 y 1975), Kaplún (1984, 1985 y 1992) y Prieto (1984, 1992 y 1998), posteriormente, permitieron sentar las bases de un modelo de comunicación-educación cuyos objetivos fundamentales se pueden concretar en: favorecer la participación, el empoderamiento y la toma de conciencia de la ciudadanía para generar procesos de transformación a nivel político, social y cultural; en palabras de Freire, para impulsar procesos de «concientización» que permitan desarrollar procesos de «politización» (1970/2007); en este sentido, entendemos la comunicación educativa desde su dimensión pedagógico-política.

Desde este marco teórico, establecemos nuestro objeto de estudio a partir del trabajo de Gamson (1992) sobre los marcos de acción colectiva y sobre la noción de cultura política, como veremos a continuación.

\section{La cultura política como objeto de estudio}

Definimos la cultura política desde el enfoque interaccionista de la teoría del comportamiento colectivo, un enfoque que tiene su origen en la Escuela de Chicago y que plantea los movimientos sociales como fuente de nuevas ideas y nuevos significados de los acontecimientos sociales y políticos (Laraña, 1996). En este sentido, la perspectiva interaccionista del comportamiento colectivo surge como respuesta a los planteamientos funcionalistas-estructuralistas y se aleja de teorías como la «movilización de recursos» o las «oportunidades políticas», más centradas en interpretar las causas que 
motivan la aparición de un movimiento social y/o en explicar las estructuras organizativas a través de las que se configura su identidad y su repertorio de acción que en comprender los procesos de producción cultural y de construcción de significados que estos colectivos llevan a cabo a través de sus acciones.

Desde la perspectiva interaccionista el tema central para el estudio de los movimientos sociales es «la aparición de nuevas construcciones de significados, procesos, normas y creencias [...]. La importancia de un movimiento [desde esta perspectiva] radica en su incidencia en los procesos de cambio social» (Gusfield, 1994, p. 96); es decir, se centra en el proceso a través del cual un movimiento social específico genera nuevos marcos para interpretar la realidad social y política. De este modo, este enfoque microsociológico sitúa la dimensión cultural de los movimientos sociales en el centro del marco analítico. A partir de estas ideas e inspirado por la teoría de los frames de Gregory Bateson - desde la perspectiva cognitiva - y de Erving Goffman — desde la perspectiva cultural—, Gamson (1992) teoriza sobre los marcos de acción colectiva y sobre la noción de cultura política para explicar la forma en la que los miembros de un movimiento social piensan y actúan sobre asuntos políticos.

Para este autor la cultura politica hace referencia al «conjunto de sistemas culturales disponibles para hablar, pensar, escribir y actuar en la política» (Gamson, 1992). La cultura política de los participantes en un movimiento social posee una estructura que Gamson denomina «paquete cultural», una estructura conformada por un conjunto de dispositivos simbólicos en cuyo núcleo se encuentra una idea central o marco que permite dar sentido a los elementos que conforman el paquete así como organizar la experiencia y orientar la acción.

En esta investigación, nos hemos servido de la noción de cultura política para definir nuestro objeto de estudio. Entendemos la cultura política desde una dimensión pedagógica tanto en cuanto a su carácter individual — la formación de la cultura política de los individuos - como a su carácter social - la formación de la cultura política de la sociedad en su conjunto-. Pretendemos analizar la formación de la cultura política de los participantes en los proyectos de comunicación surgidos a raíz del Movimiento 15-M pues, 
a nuestro juicio, el 15-M es un movimiento social claramente orientado a la transformación de la cultura política de la ciudadanía.

\section{Las prácticas asamblearias y la formación de la cultura política de la ciudadanía}

Diferentes autores coinciden en destacar el potencial simbólico y cultural del Movimiento 15-M, su impacto en la transformación de los esquemas de pensamiento social y político, y su influencia en la construcción de otros marcos de interpretación de la realidad (Barranquero y Calvo, 2013; Candón, 2013 y 2014; Cruells e Ibarra, 2013). En este sentido, como señalan Antentas y Vivas (2012), el movimiento ha generado un fuerte proceso de repolitización de la sociedad así como procesos de pedagogía política para la auto-organización y para ejercicio de la democracia directa, procesos pedagógicos colectivos que nos han permitido aprender a aprender y aprender a deshacernos de las ideas hegemónicas que configuran y enmarcan nuestra capacidad de intervención sobre la realidad social y política.

Asimismo, el Movimiento 15-M ha sido definido como un movimiento transversal que enfatiza la necesidad de generar otras formas de participación social (Laraña y Díez, 2012); con estos términos, los autores hacen referencia al rechazo explícito del movimiento hacia el sistema político bipartidista imperante en España. No obstante, estas características engloban, a nuestro entender, no sólo al sistema político entendido desde una perspectiva institucional, orgánica y/o partidista, sino que incluyen también a la cultura política entendida como una manifestación de la diversidad social, como una producción simbólica que surge de la vida pública. En este sentido, coincidimos con Barranquero (2012) cuando define al 15-M como un «movimiento de movimientos» pues participan en él, de modo transversal, diferentes edades, clases sociales, organizaciones y culturas ciudadanas.

El Movimiento 15-M, por lo tanto, se caracteriza por su grado de inclusividad, por haber sabido articular en un mismo discurso diferentes reivindicaciones y sensibilidades sociales. Desde este enfoque, la transversalidad es una forma de cuestionar nuestro modelo social y político en su totalidad, es la expresión de la 
necesidad de problematizar el espacio público para generar una toma de conciencia en la ciudadanía y para impulsar dinámicas de transformación política, social y cultural. Esta idea es la que expresaban algunas de las pancartas desplegadas por el movimiento durante la acampada en la Puerta del Sol de Madrid: «Dormíamos, despertamos» o «No nos vamos, nos mudamos a tu conciencia».

El 15-M ha tenido desde su origen una clara vocación pedagógica para la formación de la cultura política de la ciudadanía. En otro trabajo, definíamos este movimiento como un laboratorio de ideas, como un espacio de investigación-acción-participativa que ha canalizado procesos de aprendizaje y de experimentación para la construcción de otros modelos de sociedad, de otra cultura política (Barbas, 2013). De este modo, tanto el movimiento, en su totalidad, como las actividades que ha llevado a cabo, en particular, pueden ser observados desde una perspectiva pedagógico-política; las prácticas asamblearias son un claro ejemplo de ello.

Para Corsín y Estalella (2013, p. 62) la metodología asamblearia del Movimiento 15-M es «una forma de asociación a través de la cual se imagina y practica una ciudad distinta y se experimenta con nuevos modos de política». No obstante, la metodología asamblearia no es una innovación del Movimiento 15-M, es una práctica habitual de los movimientos sociales que surgieron a partir de los años 90 en diferentes partes del mundo y es, asimismo, la metodología de organización característica de los colectivos anarquistas y libertarios. El 15-M se ha nutrido de estas tradiciones académicas pero ha conseguido visibilizar las prácticas asamblearias más allá de los entornos habituales del activismo, ha hecho de ellas un lenguaje de posibilidad (McLaren, 1997) que permite ampliar los cauces de participación de la ciudadanía en los asuntos públicos y experimentar con nuevas formas de organización social y política; en palabras de Freire (1970/2007), podríamos decir que las prácticas asamblearias llevadas a cabo por el Movimiento 15-M han sido un «inédito viable» que ha permitido descubrir posibilidades de transformación cuya viabilidad no era percibida como ahora se percibe.

Como señala un miembro de Ágora Sol Radio «me acerqué a las asambleas porque estaba quemado, indignado con todo lo que estaba pasando, pero no sabía muy bien cómo canalizar eso que sentía... Entonces, vi que había gente 
de todo tipo, mayores y jóvenes, gente con formación y gente con un nivel educativo más bajo, y vi que todo el mundo aprendía hablando, intercambiando nuestro cabreo, escuchando las opiniones de gente diferente a ti, era como una escuela en la calle y eso hizo que me implicara en las asambleas».

A partir de la metodología asamblearia, el movimiento ha impulsado dinámicas de democracia deliberativa que ponen de manifiesto la necesidad de problematizar la realidad; las asambleas han sido, de este modo, tanto canales de expresión como instrumentos de construcción de otra cultura política. Del mismo modo, la metodología asamblearia ha sido aplicada en los proyectos de comunicación del movimiento; en este sentido, interpretamos dichos proyectos como entornos propicios para analizar la relación entre la comunicación educativa y los procesos de formación de la cultura política que los participantes podrían estar experimentando.

\section{Los proyectos de comunicación del Movimiento I5-M como entornos de comunicación educativa y de cultura política}

Los proyectos de comunicación del Movimiento 15-M surgieron «para generar formas propias de comunicación y crear canales de circulación libres, no sometidos a control ni de contenidos ni de ámbitos de difusión o reproducción» (De la Rubia, 2011, p. 184). Ágora Sol Radio, Adiovisol, Madrid 15-M, Fotomovimiento y Tomalatele son sólo algunos de los proyectos de comunicación creados al calor del $15-\mathrm{M}$, proyectos configurados e inspirados por los principios y por las acciones del movimiento. Cada uno de ellos responde a una serie de características específicas en función de sus formatos, de sus contenidos y de sus medios de difusión entre otros elementos. Sin embargo, estos proyectos de comunicación forman parte de las acciones que el 15-M ha desarrollado para impulsar la creación de espacios de participación que permitan profundizar en los valores cívicos que el movimiento ha promovido desde su aparición; persiguen generar procesos de concienciación crítica en la ciudadanía así como la transformación de los marcos de interpretación de la realidad social y política. 
Para el movimiento, la comunicación es un objetivo sociopolítico en sí misma (Barranquero, 2014; Candón, 2014; Marí, 2012). Tanto su modelo de organización como el tipo de contenidos y el tratamiento de la información difundida ponen de manifiesto la pretensión de llevar al ámbito mediático y comunicacional la cultura política desarrollada en las redes y en las plazas a través de la metodología asamblearia; desde esta perspectiva, los proyectos de comunicación no son sólo objetivos sociopolíticos sino que son también prácticas sociopolíticas.

Al revisar los contenidos difundidos en estos proyectos de comunicación comprobamos que suelen tratarse temáticas que tienen escasa o nula visibilidad en los medios de comunicación convencionales. Asimismo, el tratamiento de la información se realiza sin las restricciones comerciales, partidistas y/o económicas de los grandes medios, algo que permite abordar los acontecimientos sociales y políticos desde su complejidad e interdependencia. Cabe destacar, a este respecto, el tratamiento informativo realizado sobre los diversos colectivos y reivindicaciones surgidos a partir de las movilizaciones del 15 de mayo de 2011 así como la importancia otorgada a la innovación en materia de cultura, de política, de comunicación o en materia de nuevas formas de ciudadanía.

Del mismo modo, es fundamental subrayar que los procesos de producción se realizan desde la mirada poliédrica que posibilitan las estructuras horizontales y el trabajo colaborativo; en este sentido, uno de los participantes en Ágora Sol Radio señala: «Una de las cosas más importantes que he aprendido tanto del 15-M como de Ágora Sol Radio es la importancia de trabajar de forma colaborativa, parece una cosa mágica pero las cosas que surgen de la colaboración son más ricas y más potentes de lo que uno haría por su cuenta, en soledad».

Los miembros de Ágora Sol Radio manifiestan que tanto su participación en las asambleas del 15-M como su implicación en la producción de proyectos de comunicación han sido claves para la formación de su cultura política. La mayoría de los participantes expresan la importancia que tiene para ellos la participación en un medio de comunicación de estas características: «Yo, a nivel político, soy otra persona desde el 15-M y ahora, en la radio, pues... no 
paro de formarme constantemente en multitud de temas, mi cultura política ha crecido mucho y sigue creciendo».

En conclusión, los proyectos de comunicación llevados a cabo por el Movimiento 15-M pueden ser analizados desde la dimensión pedagógica-política. La aplicación de la metodología asamblearia en sus formas de organización, así como los contenidos difundidos, el tratamiento de la información y las dinámicas de trabajo colaborativo son elementos que constituyen una forma de comunicación prefigurativa a través de la que los participantes pretenden poner en práctica el modelo social y político que persiguen. De esta manera, los procesos comunicacionales podrían estar influyendo en la formación de la cultura política de los participantes.

\section{Referencias}

Antentas, J. M. y E. Vivas (2012): «El nuevo internacionalismo de la indignación», en Fernández, J. Sevilla, C. y Urbán, M. (Eds.): ¡Ocupemos el mundo! Occupy the world! (pp. 93-103): Icaria, Barcelona.

Aparici, R. y A. García Matilla (1987): Lectura de imágenes, Ediciones de la Torre, Madrid.

- (2008): Lectura de imágenes en la era digital, Ediciones de la Torre, Madrid.

Barbas, Á. (2012): «Educomunicación: desarrollo, enfoques y desafíos en un mundo interconectado», Foro de Educación. Pensamiento, cultura y sociedad, 14, pp. 157-175.

- (2013): «Volver a pensar la educomunicación», en Aranda, D. CREUS, A. y SÁnchez-NAVARRo, J. (Eds.): Educación, medios digitales y cultura de la participación, UOCPress, Barcelona, págs. 119-135.

Barranquero, A. (2012): «Redes digitales y movilización colectiva. Del 15-M a las nuevas prácticas de empoderamiento y desarrollo local», en Martínez, M. y Sierra, F., Comunicación y desarrollo. Prácticas comunicativas y empoderamiento local, Gedisa, Barcelona, pp. 377-400.

- (2014): «Comunicación, cambio social y ONG en España. Pistas para profundizar en la cultura de la cooperación desde los nuevos movimientos comunicacionales. El caso del 15M», COMMONS. Revista de Comunicación y Ciudadanía Digital, 3(1), pp. 6-28. 
Barranquero, A. y B. Calvo (2013): «Claves para comprender el 15-M: Comunicación, redes sociales y democracia deliberativa», Diálogos de la Comunicación, 86. Recuperado de: <http://dialogosfelafacs.net/ wp-content/uploads/2013/01/86_Revista_Dialogos_Claves_para comprender el 15-M.pdf $>$.

Candón, J. (2013): «Movimientos por la democratización de la comunicación: los casos del 15-M y \#yosoy132», Razón y Palabra, 82. Recuperado de: < $\underline{\mathrm{http}}$ :/www.razonypalabra.org.mx/N/N82/V82/32 Candon_V82.pdf $>$.

- (2014): «Comunicación, internet y democracia deliberativa en el 15M», en Calleja-López, A., Monterde, A., Serrano, E. y Toret, J. (Eds.), 15MP2P. Una mirada transdisciplinar del 15M, DOI: 10.7238/ in3.2014.1, pp. 107-119.

Corsín, A. y A. Estalella (2013): «Asambleas populares: el ritmo urbano de una política de la experimentación», en CRUELls, M. e IBARRA, P. (Eds.), La democracia del futuro. Del $15 \mathrm{M}$ a la emergencia de una sociedad civil viva, Icaria, Barcelona, pp. 61-79.

Cruells, M. y P. Ibarra (2013): La democracia del futuro. Del 15M a la emergencia de una sociedad civil viva, Icaria, Barcelona.

De la Rubia, R. (Ed.) (2011): Hacia una revolución mundial no violenta. Del $15 \mathrm{M}$ al 15O, Manuscritos, Madrid.

Ferrés, J. (2008): La educación como industria del deseo, Gedisa, Barcelona.

- (2014): Las pantallas y el cerebro emocional, Gedisa, Barcelona.

Freire, P. (1970/2007): Pedagogía del oprimido, Siglo XXI, Madrid.

Gamson, W. (1992): Talking Politics, Cambridge University Press, Cambridge.

GuSFIELD, J. (1994): «La reflexividad de los movimientos sociales: una revisión de las teorías sobre la sociedad de masas y el comportamiento colectivo», en LARAÑA, E. y GuSfield, J., Los nuevos movimientos sociales. De la ideología a la identidad, CIS, Madrid, pp. 93-117.

Gutiérrez, F. (1973): El lenguaje total. Una pedagogía de los medios de comunicación, Humanitas, Buenos Aires.

- (1975): Pedagogía de la comunicación, Humanitas, Buenos Aires

Hernández, E.; M. C. Robles y J. B. Martínez (2013): Jóvenes interactivos y culturas cívicas: sentido educativo, mediático y político del $15 \mathrm{M}$. Comunicar, 40, pp. 59-67. DoI: 10.3916/C40-2013-02-06.

KaPlún, M. (1984): Comunicación entre grupos: el método del Cassette-Foro, 
Centro Internacional de Investigaciones para el Desarrollo, Bogotá.

- (1985): El comunicador popular, CIESPAL/CESAP/RADIO NEDERLAND, Quito.

— (1992): A la educación por la comunicación, CIESPAL, Quito.

LARAÑa, E. (1996): «La actualidad de los clásicos y las teorías del comportamiento colectivo», Reis: Revista española de investigaciones sociológicas, 74, pp. 15-44.

LARAÑa, E. y R. DíEz (2012): «Las raíces del Movimiento 15-M. Orden social e indignación moral», Revista Española del Tercer Sector, 20, pp. $105-144$.

MARí, V. M. (2012): Conectados, endeudados, indignados. Un análisis comunicacional del Movimiento 15-M, Foro Ignacio Ellacuría, Murcia.

Masterman, L. (1993): La enseñanza de los medios de comunicación,Ediciones de la Torre, Madrid.

McLaren, P. (1997): Pedagogía crítica y cultura depredadora. Políticas de oposición en la era posmoderna, Paidós, Barcelona.

McQuaIL, D. (1999): Introducción a la teoría de la comunicación de masas, Paidós, Barcelona.

Porcher, L. (1974): La escuela paralela, Kapelusz, Buenos Aires.

Prieto, D. (1984): «Apuntes sobre la comunicación popular educativa», Cuadernos de Chasqui. Revista de Latinoamericana de Comunicación, 2. Recuperado de: $<$ http://repositorio.ciespal.org:8080/bitstream/123456789/157/2/CIESPAL $\% 20$ Monograf $\% \mathrm{C} 3 \%$ ADas $\% 20$ 3\%20PUBCOM.pdf>.

- (1992): «Comunicación y educación», Cuadernos de Chasqui. Revista de Latinoamericana de Comunicación, 8. Recuperado de: $<$ http://186.5.95.155:8080/bitstream/123456789/187/2/CIESPAL\%20Daniel\%20Prieto\%20C.pdf $>$.

- (1998): «Comunicación educativa en el contexto latinoamericano», Intervención Psicosocial, 7(3), pp. 329-345.

VAllet, A. (1977): El lenguaje total, Luis Vives, Barcelona. 